\title{
Body Weight Gain Result Standard Unit
}

National Cancer Institute

\section{Source}

National Cancer Institute. Body Weight Gain Result Standard Unit. NCI Thesaurus. Code C119776.

The standard unit of measure for body weig ht gain results. 